\title{
Prescription Audit in an Outpatient Pharmacy of a Tertiary Care Teaching Hospital-A Prospective Study
}

\author{
Deepa Sunny ${ }^{1,2, *}$, Kalyan Roy², Sherin Sara Benny ${ }^{1}$, Dano Chamakalayil Mathew ${ }^{1}$, Jarupala Gangadhar Naik ${ }^{1}, K^{2}$ arunakaran Gauthaman ${ }^{2}$ \\ ${ }^{1}$ Karavali College of Pharmacy, Vamanjoor, Karnataka, INDIA. \\ 2Department of Pharmacology, Himalayan Pharmacy Institute, Majhitar, Rangpo, East Sikkim, INDIA.
}

\begin{abstract}
Background: Prescription audit aids in evaluating the quality of medical treatment offered to the patients. It helps to discern any effective changes that would support health care professionals to proffer superior quality of care to the patients. Aim of the study is to carry out prescription audit in the outpatient pharmacy department of a tertiary care teaching hospital. Methods: A prospective observational study was conducted on 500 prescriptions for a span of four months in the outpatient pharmacy department of a tertiary care teaching hospital in South Karnataka, India. All the prescriptions were analyzed based on WHO prescribing indicators and were evaluated for errors in prescription writing. Data were entered and analyzed using into SPSS. Graphic representation has been used for visual interpretation of the analyzed data. Results: Five hundred scripts comprising of 1,661 drugs were analyzed. The average number of drugs per prescription was three. The study encompassed $52 \%$ males and $48 \%$ females. Most patients were from the age group of $41-60$ years. Only $3.6 \%$ (18) of medications were prescribed by generic names. Patients received 9\% (145) medications contained in Essential Drug List and prescriptions containing antibiotics were $19 \%$ (97). Majority of the prescriptions were from Orthopaedics (18.6\%) tailed by General Medicine (15.8\%). Consecutively
\end{abstract}

Analgesics $(12.7 \%)$ were the most commonly prescribed drug class, among which Diclofenac and Paracetamol+Tramadol were usually prescribed, this was tailed by gastrointestinal medicines (11.7\%). Conclusion: Prescription audit can be helpful to plan appropriate intervention to ensure the rational drug therapy and to evaluate the existing drug use pattern. It also reflects the perspectives of current prescribing pattern in hospitals.

Key words: Prescription audit, WHO prescribing indicator, Essential drugs, Outpatient Pharmacy.

Correspondence

Dr. Deepa Sunny,

Department of Pharmacology, Himalayan Pharmacy Institute, Majhitar-737136, Rangpo, East Sikkim, INDIA.

Phone: +91 8281461650

Email: deepsunny93@gmail.com

DOI: 10.5530/jyp.2019.11.85

\section{INTRODUCTION}

An audit in healthcare is a system used by health professionals to determine, appraise and improve the care of patients in an efficient way and it measures existing practice against a defined standard. ${ }^{1}$ Audit facilitates in the assessment of the contemporary trends of drug handling, drug disbursement, appropriateness of prescriptions and adherence to evidence-based recommendations. ${ }^{2}$

On examining a prescription it can be differentiated into an intellectual and technical part. The intellectual segment involves decision making, which encompasses knowledge of diagnosis, interactions and contraindications. The technical section involves imparting of essential information such as, drug name and dose to the pharmacist. ${ }^{3}$

\section{MATERIALS AND METHODS}

A Prospective and observational study was undertaken in the Outpatient pharmacy of a tertiary care teaching hospital. An assessment of 500 prescriptions was done over a span of four months from October 2016 to January 2017. Prior to conduct of study, Ethical clearance was obtained from the Institutional Ethics Committee of Father Muller Medical College Hospital, Mangalore. (FMMC/FMIEC/3052/2016). All the prescriptions presented to outpatient pharmacy between 10 am to $1: 30 \mathrm{pm}$ and patients of all age group were included in the study. Prescriptions presented by admitted patients, prescriptions containing only one drug and prescriptions from casuality/EMG and Dental department were excluded.

\section{Source of Data}

The data source required to conduct the study was retrieved from the patient's prescription presented at the outpatient pharmacy. Analysing errors in prescription writing, Patient demographic data analysis, WHO prescribing indicator, Number of prescriptions from the different department, commonly prescribed class of drugs, commonly prescribed dosage form and Drug-Drug Interactions.

\section{Statistical Analysis}

Data were entered into SPSS and analysis was done. Descriptive statistics such as frequencies and percentages were calculated for categorical variables. Graphic representation has been used for visual interpretation of the analyzed data.

\section{RESULTS}

An appraisal of 500 prescriptions was done to analyze the demographic details presented in the prescription in conjugation with the scripts for the legibility and the use of non-standard abbreviations. The evaluated prescriptions contained a sum of 1661 drugs.

Out of 500 prescriptions patient name and OP number were present in 498 (99.6\%) and 493 (98.6\%) of the prescriptions correspondingly. The name and signature of the prescriber were present in 485 (97\%) and 476 (95\%) of the prescriptions. Legibility was 456 (91\%) in the prescriptions analyzed for the study, these prescriptions were clear and understandable while the remaining were illegible. The prescriptions entailed a total of

This is an open access article distributed under the terms of the Creative Commons Attribution-NonCommercial-ShareAlike 4.0 License, which allows others to remix, tweak, and build upon the work non-commercially, as long as the author is credited and the new creations are licensed under the identical terms. 
15 error-prone abbreviations and $84 \%$ of the scripts were written in capital letter.

\section{Patient Demographics}

The demographics that is the percentage of male and female as well as the percentage of each age group were calculated. Out of 500 prescriptions, we encountered a greater number of male patients $261(52.2 \%)$ than female patients 239 (47.8\%).

While considering the age, we divided the patient into four age groups, most of the prescriptions hailed from the age group 41-60 years (196 scripts) followed by age group 21-40 years (126 scripts). Details are given in Table 1.

\section{Analysis of WHO core prescription indicators}

The prescriptions were analyzed in accordance with WHO Core Prescribing indicators and the result were as follows, average number of drugs per prescription was three which tallies with the ideal value $(\leq 3)$. Percentage of encounters with an antibiotic was $19.4 \%$ and this is within the WHO ideal value $(\leq 30)$ thus indicating the rational use of antibiotics. Percentage of encounters with injection was found to be $3.6 \%$ which is also within the WHO ideal value $(\leq 10)$. Only $3.6 \%$ of drugs were prescribed by generic name. Percentage of drugs prescribed from EDL (Essential Drug List) was $9 \%$. The drugs were paralleled to the National List of Essential Medicine 2015 and only 145 drugs were found to be from this list. According to WHO the ideal value of generic and drugs from EDL should be $100 \%$. Following Table 2 shows the comparison of individual indicators between study group and WHO- Ideal.

\section{Department wise analysis of prescriptions}

500 prescriptions were categorized based on patient's visit to a particular department. The highest number of prescriptions were from Orthopaedics (18.6\%) followed by General Medicine (15.8\%). Particulars are shown in Figure 1.

\section{According to Dosage form}

The percentage of FDCs in the form of tablet and capsule was 36\%. With regard to dosage form, it was found that greater number of medicines were prescribed per oral $(81.2 \%)$, the oral dosage form consists of tablets $69.47 \%$, capsules $(5.71 \%$ ) and syrup $6.02 \%$, followed by Topical preparations, particulars are shown Figure 2

\section{Number of drugs per prescription}

Number of Drugs prescribed in prescription is indication for checking the polypharmacy, in this study prescription containing only one drug had been excluded. Out of 500 prescriptions majority of scripts contained three drugs in 226 prescriptions (45\%) followed by four drugs in 125 prescriptions $(25 \%)$.

\section{Commonly prescribed class of Drug}

The most commonly prescribed class of drug was found to be Analgesics (13\%) followed by GIT drugs (12\%), CVS drugs (11\%) and the least

Table 1: Number of prescriptions from different age groups.

\begin{tabular}{cccc}
\hline Sl. no & Age & $\begin{array}{c}\text { Number of Prescriptions } \\
(\text { No=500) }\end{array}$ & Percentage (\%) \\
\hline 1 & $0-20$ & 56 & 11.2 \\
2 & $21-40$ & 126 & 25.2 \\
3 & $41-60$ & 196 & 39.2 \\
4 & $>60$ & 122 & 24.4 \\
\hline
\end{tabular}

Table 2: Comparison of individual indicators between study group and WHO- Ideal.

\begin{tabular}{ccc}
\hline Prescribing Indicators & Study result & Ideal value \\
\hline $\begin{array}{c}\text { Average number of drugs per } \\
\text { prescription }\end{array}$ & 3.32 & $\leq 3$ \\
$\begin{array}{c}\text { Percentage of encounters with an } \\
\text { antibiotic }\end{array}$ & 19.4 & $\leq 30$ \\
Percentage of encounters with an \\
$\quad \begin{array}{c}\text { injection } \\
\text { Percentage of drugs prescribed by } \\
\text { generic name } \\
\text { Percentage drugs prescribed from } \\
\text { EDL }\end{array}$ & 3.6 & 100 \\
\hline
\end{tabular}

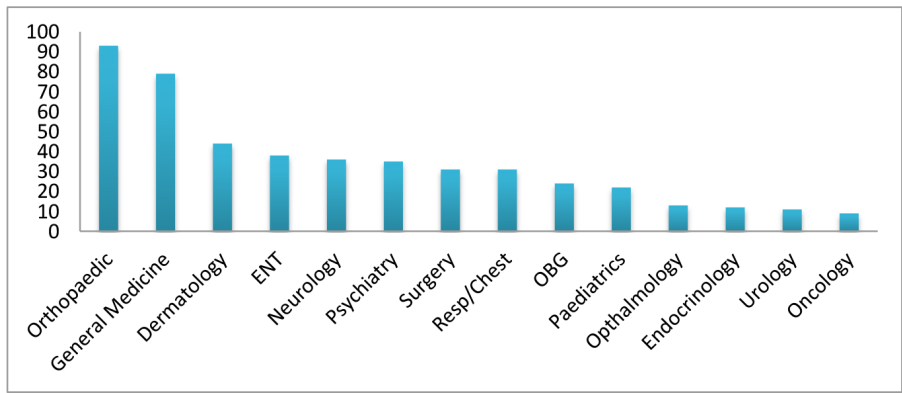

Figure 1: Number of prescriptions from different OPD.

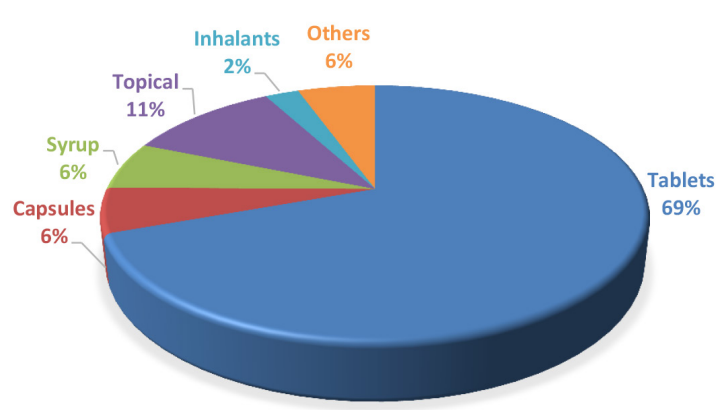

Figure 2: Prescribed drug dosage form.

prescribed class of drug was Endocrinological medicines (4\%). Out of $13 \%$ of the Analgesics the most prescribed medication was Diclofenac(67) this value is also inclusive of topical diclofenac followed by a combination of Paracetamol+Tramadol(30) and Paracetamol alone (30). Out of $12 \%$ of gastrointestinal drugs most prescribed medication was Rabeprazole (57) followed by Pantoprazole (50) and a combination of Rabeprazole+Domperidone(24). Cardiovascular drugs that was prescribed commonly includes a combination of Aspirin+Atorvastatin (23), Amlodipine (14) and Telmisartan (9), particulars are shown in Figure 3.

\section{Drug-Drug Interaction}

The prescriptions were also studied for possible drug-drug interactions, we found a total of 253 Drug-Drug interactions among these were 17 Major, 208 Moderate and 28 Minor Drug-Drug interactions. Details of the possible Major Drug-Drug interactions are shown in the following Table 3. 


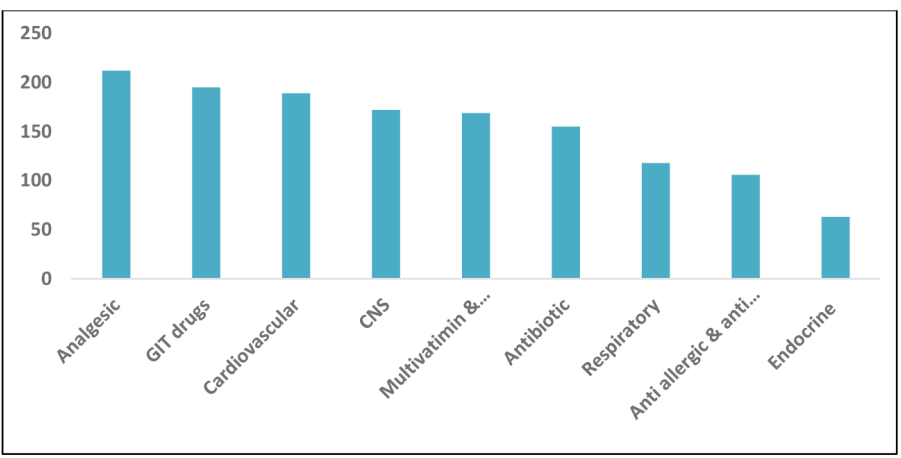

Figure 3: Commonly prescribed drug class.

\section{DISCUSSION}

Medical audit is the critical assessment of medical and healthcare related system with a vision to elicit indispensable enhancement in the quality of healthcare delivered to the patients. ${ }^{4}$ The prescribing habits are of critical importance since the therapeutic efficacy and safety solely lies in rationality of the prescriptions. In this study a sum of 500 scripts were appraised which gave an aggregate of 1661 drugs.

While investigating the errors in prescriptions, patient's detail such as, name was not included in $2(0.4 \%)$ scripts and the OP number assigned was not present in $7(1.4 \%)$ prescriptions. This result surpasses the study conducted by Gupta $\mathrm{A}^{5}$ et al.

Prescriber's name and signature was not present in 15 (3\%) and 24 (5\%) of the scripts respectively. This value is negligible when compared to study conducted by Sirisha ${ }^{6}$ et al. The legibility of the assessed scripts was $91 \%$, this is comparable to the studies done by Nagashree $\mathrm{BN}^{7}$ et $\mathrm{al}$. and Balbir $^{8}$ et al.

We encountered a higher number of male cases (52.2\%) than the female cases $(47.8 \%)$. Our study result corresponds to that conducted by Darji ${ }^{9}$ et al. Most of the scripts were from the age distribution of 41-60 years (196 scripts), followed by $21-40$ years (126 scripts).This is in contrast to the study conducted by $\mathrm{Abidi}^{10}$ et al. in which frequent prescriptions were from the age group of children ( $\leq 14$ years)followed by adolescents (15-19 years).

The average number of drugs per prescription was three, this tally with the $\mathrm{WHO}^{11}$ ideal value $(\leq 3)$. The reasoning sought behind this is that when more drugs are prescribed there is increased possibility of drug interactions and patient non-compliance. The percentage of drugs prescribed by generic name was only $3.6 \%$, which is a far diminished value when compared to the study conducted by Srividya ${ }^{12}$ et al. and the WHO ideal (100\%). The use of generic drugs dissipates the cost of healthcare as well as permits the patient to play a more active role in identifying the prescribed medicines. The percentage of encounters with an antibiotic prescribed was $19.4 \%$, which is within the limit set by WHO $(\leq 30 \%)$. This is an evidence of the rational usage of antibiotics in the hospital, this practice should constantly be encouraged through educational interventions. Drugs ordered based on EDL was found to be $9 \%$, which is lower than the WHO standard (100\%). The percentage of encounters with an injection prescribed was $3.6 \%$, which is within the limit set by WHO $(\leq 10)$. Since this study was carried out in the outpatient pharmacy the use of injections was likely to be less.

The primarily prescribed drug class observed was Analgesics (13\%), followed by GIT drugs (12\%) and CVS drugs (11\%). The study result corresponds to that carried out by Gupta $\mathrm{A}^{5}$ et al. In the present study, majority of the prescription belongs to Orthopaedics (18.6\%) followed by General Medicine department $(15.8 \%)$ the result contradicts the
Table 3: Possible Major Drug-Drug Interactions found in this study.

\begin{tabular}{|c|c|}
\hline Interaction & Effect \\
\hline Enalapril + Spironolactone & $\begin{array}{l}\text { Increased risk of Hyperkalaemia on } \\
\text { concomitant administration of ACE } \\
\text { inhibitors and potassium sparing diuretics. }\end{array}$ \\
\hline Lorazepam + Olanzapine & $\begin{array}{l}\text { Cardiorespiratory depressant effect } \\
\text { on concomitant use of olanzapine and } \\
\text { benzodiazepines especially in the elderly and } \\
\text { debilitated patients. }\end{array}$ \\
\hline
\end{tabular}

Amitriptyline + Topiramate

Central nervous depressant effects, alteration in fluid and electrolyte balance, inhibition of peripheral sweating mechanism, this in turn affecting adjustment to temperature changes.

Theophylline + Nebivolol

Nonselective and high doses of cardio selective beta-blockers may cause fatal bronchospasm by opposing theophylline induced bronchodilator.

Theophylline + Tramadol

Increased risk of seizures during co administration of tramadol.

Warfarin + Aspirin

Propranolol + Theophylline

Additive anticoagulant effect.

Non-selective and high doses of cardio selective beta-blockers may cause fatal bronchospasm by opposing theophylline induced bronchodilator.

Propranolol + Formoterol

Beta -blockers may antagonize the effects of beta- 2 adrenergic bronchodilators and precipitate acute, life threatening

bronchospasm in patients with asthma or other obstructive airway diseases.

Tramadol + Levofloxacin

Increased risk of seizures during co administration of tramadol.

HCQ + Leflunomide

Coadministration of Leflunomide with other immune- or myelosuppresiveantirheumatic agents may potentiate the risk of infections.

Budesonide + Leflunomide Coadministration of Leflunomide with other immune- or myelosuppresiveantirheumatic agents may potentiate the risk of infections.

Amitriptyline + Tramadol

Spironolactone + Telmisartan

Increased risk of seizures during co administration of tramadol.

(found in 2 scripts)

Clonazepam + Olanzapine

Increased risk of Hyperkalaemia on concomitant administration of ARBs and potassium sparing diuretics.

Cardiorespiratory depressant effect on concomitant use of olanzapine and benzodiazepines especially in the elderly and debilitated patients.

Spironolactone + Ramipril

Increased risk of Hyperkalaemia on concomitant administration of ACE inhibitors and potassium sparing diuretics.

Nortryptyline + Tramadol

Increased risk of seizures during co administration of tramadol with any substance that can reduce seizure threshold.

study done by Bandyopadhyay ${ }^{4}$ et al. where majority of patients were from general medicine, followed by Paediatrics.

The percent of FDCs in the dosage form of tablet and capsule was $36 \%$. The prescriber selects a FDC when the efficacy of combinational medicament outweighs that of an individual entity. In regard to the dosage form, it was evident that greater number of medicines were prescribed per oral (81.2\%) followed by Topical preparations (11\%) and 
inhalants (2\%). The oral dosage form consists of tablets $69.47 \%$, capsules (5.71\%) and syrup $6.02 \%$, The dosage form opted for is hinged on optimizing patient medication therapy. Furthermore, it is influenced by the medical condition of the patient and the drug's chemical stability and pharmacokinetics.

The majority of the prescriptions contained three drugs in 226 prescriptions (45\%) followed by four drugs in 125 prescriptions (25\%), this is consistent to the study by Farnoud ${ }^{13}$ et al. The study result is suggestive of no polypharmacy.

While analyzing the scripts for possible drug-drug interactions, an astonishing probability of encountering 253 drug-drug interactions was interpreted. A larger proportion of interactions were moderate (208) trailed by minor interactions (28). Drug interactions can be potentially hazardous but most of the consequences of interactions could be overcome with careful monitoring of the patient and their drug therapy.

\section{CONCLUSION}

Based on the results of audit it can be concluded that prescription writing is apprpached mindfully and there is no irrational use of drugs. With the help of such audit medical professionals especially the prescribers become more aware of the current practices and would drive themselves to safe, economic and effective therapeutic practice.

\section{ACKNOWLEDGEMENT}

The authors thank Dr. Ravi Kumar Nayak, Professor, Karavali College of Pharmacy for providing all the necessary assistance required for the study and also his moral support.

\section{CONFLICT OF INTEREST}

The author declare that they have no conflict of interest

\section{ABBREVIATIONS}

EMG: Emergency; WHO: World Health Organisation; GIT: Gastrointestinal Tract; CVS: Cardiovascular system; FDC: Fixed Dose Combination; OP: Out Patient; HCQ: Hydroxychloroquine.

\section{REFERENCE}

1. Esposito P. Clinical audit, a valuable tool to improve quality of care: General methodology and applications in nephrology. World J Nephrol. 2014;3(4):249.

2. Hussain S, Parveen Z, Gupta S, Kumar D, Gupta R, Thakur S. A study of prescription auditing in rural health care setting of north India. Int J Med Sci Public Heal. 2016;5(12):2461.

3. Marianne L, Lars PNJM. Errors in the medication process: Frequency, type and potential clinical consequenses. Int J Qual Heal Care. 2005;17(1):15-22.

4. Bandyopadhyay D, Banerjee NC, Chattopadhyay S, Singha P. A Study of Prescription Auditing in a Tertiary Care Teaching Hospital of Eastern India. J Drug Deliv Ther. 2018;4(1):140-9.

5. Gupta A, Mishra S. Prescription audit study in a tertiary care hospital using the anatomical therapeutic chemical and defined daily dose classification concept. Int J Basic Clin Pharmacol. 2014;3(5):889.

6. Sirisha S, Thomas SM, Varghese A, Reddy R, Baby B, Gudur SP. A Descriptive study on prescription audit in india-A REVIEW. Indo American Journal of Pharmaceutical Sciences. 2015;2(3):641-7.

7. Nagashree BN, Manchukonda RS. Prescription audit for evaluation of present prescribing trends in a rural tertiary care hospital in South India: An observational study. International Journal of Basic and Clinical Pharmacology Research. 2016;5(5):2094-7.

8. Balbir K, Rani W. Prescription audit for evaluation of prescribing pattern of the doctors for rational drug therapy in a tertiary care hospital. J Drug Deliv Ther. 2013;3(5):77-80

9. Darji N, Vaniya H, Doshi C, Hedamba R, Jadav S, Trivedi H. Prescription audit in the inpatients of a tertiary care hospital attached with medical college. J Clin Exp Res. 2016;3(2):197.

10. Abidi A, Gupta S, Kansal S, Ramgopal R. Prescription auditing and drug utilization pattern in a tertiary care teaching hospital of western UP. Int J Basic Clin Pharmacol. 2012;1(3):184

11. World Health Organization. How to investigate drug use in health facilities. 1993;1-92. Available from: http://apps.who.int/medicinedocs/pdf/s2289e/ s2289e.pdf

12. Srividya BP, Shashikumar NS, Amardeep G. Retrospective audit of prescription of drugs among inpatients of orthopedic wards at medical college teaching hospital, Mandya. Nat J Physiol Pharm Pharmacol. 2016;6(4):282-5.

13. Farnoud G, Maryam SHS. Audit of Prescriptions in a Tertiary Care Hospital - A Retrospective Study. World J Pharm Pharm Sci. 2016;5(10):886-94.

Article History: Submission Date : 19-07-2019; Revised Date : 24-09-2019; Acceptance Date : 01-10-2019.

Cite this article: Sunny D, Roy K, Benny SS, Mathew DC, Naik GJ, Gauthaman K. Prescription Audit in an Outpatient Pharmacy of a Tertiary Care Teaching Hospital-A Prospective Study. J Young Pharm. 2019;11(4):417-20. 